\title{
Children's compliance as a function of type of instructions and payoff for noncompliance*
}

\author{
WILLIAM H. REDD, DONALD L. AMEN, TERRY D. MEDDOCK, and ANDREW S. WINSTON \\ University of Illinois at Urbana-Champaign, Champaign, Illinois 61820
}

\begin{abstract}
The role of reinforcement loss and instruction type (positive and negative ) in cinildren's compliance with adult instructions was studied using a 2 by 2 factorial design. During daily sessions 32 first- and second-grade children played a two-choice discrimination game in which one response earned two tokens (redeemable for toys) and the other earned eitner one or 0 tokens (dejending upon the group to which Ss were assigned). On successive trials either one or both responses were available. When botn responses were available, the child nad to choose between them. After $\mathrm{S}$ showed a preference for the response that paid higher magnitude reinforcement, the adult entered and gave either positive ("I want you to ...," "Do X") or negative ("I don't want you to ...," "Don't do Y") instructions. After toese instructions E left and $S$ played alone. Compliance witil eitner instruction involved S's choosing the lower paying response. Results snowed that the adult's negative instructions were more effective than positive instructions, regardless of whetner compliance meant the child's forfeiting one or two tokens. Boys were significantly more compliant than girls. Results are discussed in terms of their relevance to resistance to temptation.
\end{abstract}

The degree to which adult instructions will control children's behavior depends on a number of contextual factors. In some situations seemingly subtle differences in the instructional cues can have striking effects. The explicitness with which a prohibited response is identified (Moore \& Olson, 1969), the presentation of conflicting instructions from another adult (Stouwie, 1971), and the specific reason an adult gives for not touching a prohibited toy (e.g., LaVoie, 1973), have been found to alter children's compliance. The manner in which an adult communicates his preferences is also important. For example, Redd and Winston (1974) found that an adult's comments made about a model's performance were more effective in controlling an observing child's subsequent behavior when expressed negatively (i.e., "I don't like it when he does X") than when expressed positively (i.e., "I like it when he does Y').

Another factor that is likely to determine the effect of instructions is the cost involved in complying, i.e., how much the child loses by following instruction. In a resistance to temptation situation, Dmitruk (1971) found that children were more likely to emit a prohibited response (i.e.,cheat) if the incentive was the child's most preferred rather than least preferred toy. Unfortunately, it may be difficult to vary incentive value when the dimension cannot be readily quantified.

\footnotetext{
*This paper is sponsored by Keith C. Scott, who takes full editorial responsibility. The research $w$ as supported by a faculty grant from the National Institute of Education and by Grant 1R03 MH 24614-01A1 MSM from the National Institute of Mental Health. The authors wish to thank Janette $H$. Harris for her comments on the manuscript. Thanks also goes to T. Melohn and W. McNealy of the Philo and Unit 7 (Illinois) school systems and to $D$. Holste of the Urbana, Illinois school department for their cooperation. Reprints may be obtained from William $\mathrm{H}$. Redd, Psychology Department, Children's Research Center, University of Illinois, Champaign, Illinois 61820 .
}

Moreover, asking a child to state a preference for one toy vs another may be an unreliable method for determining the reinforcing value of stimuli. It would therefore be desirable to have a method for explicitly manipulating the incentive value of stimuli so as to be able to investigate its role in controlling behavior.

The purpose of the current study was to examine the effect of the type or form of instruction and the explicit pay-off for compliance. Children were first trained in a two-choice color discrimination task in which one response produced greater token reinforcement than the other. After a child had developed a preference for the higher paying response, the adult told him to emit the lower paying response. Instructions were stated either positively (i.e., "I want you to do X") or negatively (i.e., I don't want you to do Y."). The effect of these instructions was studied under two pay-off conditions: children lost either one or two redeemable tokens by complying.

It was predicted that negative instruction would be more effective than the positive instruction in controlling subsequent choice behavior. Second, it was expected that noncompliance would increase as the reinforcement for compliant responding decreased.

\section{METHOD}

\section{Subjects and Design}

Thirty-nine first- and second-grade children from the Urbana and Philo, Illinois school system participated as subjects. Six children were terminated for failure to reach a specified discrimination criterion during the initial phase of the experiment, and another was excluded because she was unable to identify colors of the lights used during the task. The remaining 32 subjects were distributed with five girls and three boys in each of the four cells of a 2 by 2 (instruction type by magnitude 
of reinforcers) factorial arrangement. Groups were balanced with respect to age.

\begin{abstract}
Apparatus
The apparatus consisted of a two-choice marble drop game placed on a small table, and a token dispenser located adjacent to the game. Marbles could be dropped into two holes located side-by-side on top of the apparatus. These holes were illuminated by different colored 7.5-W Christimas tree bulbs. An additional white light was illuminated whenever the experiment was in progress. Following a discrete trial procedure, one or both lights were presented on single-color and choice trials, respectively. An assistant seated behind a one-way mirror controlled the apparatus and recorded the child's responses. Two reliability checks using a second observer yielded $100 \%$ agreement.
\end{abstract}

\section{Procedure}

An assistant first showed subject a variety of toys and candies. Subject was told that he could play a game in which he could win tokens that could be traded for the prizes. Subject was then introduced to experimenter, an adult male. Experimenter led subject into the experimental room, seated him in front of the apparatus, and gave the following instruction:

"Here is a game I think you'll like to play. When a light comes on, pick up a marble and put it in the lighted hole. When you get tokens, put them in this container. Later you can take your tokens and use them to buy some of the toys you just saw."

Four single-color demonstration trials were presented. During these trials experimenter prompted subject to identify the color of the light and the number of tokens he received. One-half of the subjects received one token and the other hald received zero tokens for the low-magnitude reinforcement color. The high-magnitude reinforcement color yielded two tokens for all subjects. Experimenter then excused himself and subject played alone for 36 trials. This series consisted of 24 single-color trials with 12 choice trials randomly interspersed. Left-right position of the colors was counterbalanced.

Experimenter reentered and four more demonstration trials were presented. As before, subject was asked to identify the colors and pay-offs. The following instructions were then given:

"I have some work to do and won't be back, so you go ahead and keep playing. When this light goes out, (Experimenter indicates the white light) it means the game is over. Then take your tokens to the lady (man) outside and you can use them to buy toys. OK? I'll see you tomorrow. Bye-Bye."

After the experimenter left, 24 choice trials were presented. At the end of the session, the assistant helped the child count and trade in the tokens. These procedures were repeated each session until the child chose the color paired with greater token reinforcement significantly $(\mathrm{p}<.01)$, more than chance in the final 24 choice trials. If subject did not achieve this criterion within five sessions, he was terminated. When subject achieved the preference criterion, the instructional manipulation was carried out during the following session. Immediately preceding the final 24 choice trials, experimenter gave the usual instruction, stated that he would not return, and added the following instruction:

"Now remember ___ (Subject's name) I want (or don't want) you to put the marble in the _-colored hole. Put (or don't put) it in the -colored one."

Half the subjects received the positive instructions to put the marbles in the hole paired with low-magnitude reinforcement (one or zero tokens). The other half received the negative instruction, i.e., they were told not to put marbles in the hole paired with high-magnitude reinforcement (two tokens). Subjects behavior during the 24 subsequent choice trials served as the test of instructional control.

\section{RESULTS}

Subjects who received two vs one tokens took significantly longer to reach criterion than subjects who received two vs zero tokens $(\mathrm{F}(1,28)=13.46, \mathrm{p}<.01)$. However, subjects who received positive instructions did not differ from subjects who received negative instructions in terms of sessions to criterion $(F<1)$, and the effect of sex of subject was also nonsignificant $(\mathrm{F}<1)$.

Of major interest was subjects choices following negative and positive instructional comments. The dependent measure used in the analysis was the percent of choices (out of 24) that deviated from experimenter's instructions (i.e., percent high-magnitude choices). A 2 by 2 by 2 (Instruction Type by Magnitude of Token Reinforcement by Sex of Subject) analysis of variance (unweighted means, Winer, 1962) yielded significant main order effects for instruction type and sex of subject and no significant effect for magnitude of reinforcement. The significant effect of instruction type $(F(1,24)=15.80, p<.001)$ revealed greater percent deviant responses for subjects who received positive instructions $(M=62 \%)$ than for subjects who received negative instructions $(M=17 \%)$. The significant effect of sex of subject $(F(1,24)=5.63, p<.05)$ indicated greater percent deviant responses for girls $(M=53 \%)$ than for boys $(M=26 \%)$. The interaction of Instruction Type by Sex of Subject was not significant $(F<1)$, nor was the three-way interaction with sex of subject as a factor $(F(1,24)=1.73, p<.20)$. It should be noted that there was no tendency for instruction type or magnitude of reinforcement to interact with sex of subject separately in the two-way interaction $(\mathrm{F}<1)$.

\section{DISCUSSION}

Children showed a high degree of compliance with the adult's negative instructions not to choose the response that earned two redeemable tokens, even when complying meant that they received no token reinforcement. However, the children showed less compliance when positive instructions were used. These results were independent of the absolute magnitude of the lower-paying response. In addition, boys showed greater compliance than girls.

The greater control exerted by negative instructions is congruent with the results obtained by Redd and Winston (1973). This finding is also directly relevant to several studies of the relative effectiveness of positive and negative feedback in facilitating children's learning (i.e., that negative feedback is more effective) (Buss \& Buss, 1956; Paris \& Cairns, 1972; Spence, 1966). As Paris and Cairns pointed out, the critical task is to determine how negatively expressed instructions, comments, and feedback gain superior control in the child's natural environment. In a classroom setting, they found that teachers dispensed negative feedback much less often and much more contingently than positive feedback. Thus, reasoning from their observations, positive adult responses may not be a discriminative stimulus for any specific outcome, whereas negative feedback statements may be reliable signals to which the child attends and responds accordingly. However, it remains 
to be determined whether positive and negative instructions have also been associated with similar conditions in children's natural environments.

The fact that the children complied with the adult's negative instruction at both pay-off levels may indicate that the negative instructions exerted stronger control than originally thought. This result, in addition to the lack of control of the positive instructions at both pay-off levels, clearly indicates the superior control exerted by the negative instructions. From Dmitruk's (1971) and Winston and Redd's 1973) research, it would be predicted that the pay-off for the deviant response could be increased to the point where it would effectively compete with the negative instructions so that subjects would cease to comply. Likewise, under conditions of equal reinforcement for the two responses one would expect compliance with positive instructions to increase.

The procedures used in the current study of instructional control are similar to procedures traditionally used to study resistance to temptation. In both cases, the child must forego a more attractive alternative in order to comply with the adult's instructions. The main difference between research on instructional control and resistance to temptation appear to be the presence of an adult after the instructions are given. The adult remains present in most studies of instructional control, but is absent in studies of resistance to temptation. If resistance to temptation is viewed as a situation in which the reinforcement value of the temptation is in competition with adult instructions for control of the child's behavior, then the two areas mesh.

Thus, the current procedure of pitting instructions and prohibitions against contingencies of token reinforcement may be used in the study of resistance to temptation. This approach has the advantage of allowing the attractiveness or reinforcement value of the temptation to be functionally determined before the experimental manipulations are instituted. If the child fails to develop a clear preference for the high magnitude reinforcement alternative, the pay-offs can be easily altered, as redeemable tokens are used. Another advantage of the procedure is that the relative strength of different instructions can be examined. As in present study, different instructions can be presented in competition with different amounts of token reinforcement and their effects compared. Thus, this procedure provides a practical method of exploring the variables controlling children's resistance to temptation.

\section{REFERENCES}

Buss, A. H., \& Buss, E. H. The effect of verbal reinforcement combinations on conceptual thinking. Journal of Experimental Psychology, 1956, 52, 283-287.

Dmitruk, W. M. Incentive preference and resistance to temptation. Child Development, 1971, 42, 625-628.

LaVoie, J. C. The effect of type of punishment on resistance to deviation. Paper presented at the biennial meeting of the Society for Research in Child Development, Philadelphia, March 29, 1973.

Moore, S. G., \& Olson, F. The effects of explicitness of instructions on the generalization of a prohibition in young children. Child Development, 1969, 40, 945-949.

Paris, S. G., \& Cairns, R. B. An experimental and ethological analysis of social reinforcement with children. Child Development, 1972, 43, 71 7-729.

Redd, W. H., \& Winston, A. S. The role of antecedent positive and negative comments in the control of children's behavior. Child Development, 1974, 45, 540-546.

Spence, J. T. Verbal discrimination performance as a function of instructions and verbal-reinforcement combination in normal and retarded children. Child Development, 1966, 37, 269-281.

Stouwie, R. J. Inconsistent verbal instructions and children's resistance-to-temptation behavior. Child Development, 1971, 42, 1517-1531.

Winer, B. J. Statistical principles in experimental design. New York: McGraw-Hill, 1962.

Winston, A. S., \& Redd, W. H. Adult presence versus reinforcement contingencies in the control of children's choice behavior. Paper presented at the biennial meeting of the society for Research in Child Development, Philadelphia, 1973.

(R eceived for publication August 29, 1974.) 